\title{
Incompleteness in the Bell theorem using non-contextual local realistic model
}

\author{
Koji Nagata, ${ }^{1}$ Tadao Nakamura, ${ }^{2}$ and Han Geurdes ${ }^{3}$ \\ ${ }^{1}$ Department of Physics, Korea Advanced Institute of Science and Technology, Daejeon 34141, Korea \\ E-mail: ko_mi_na@yahoo.co.jp \\ ${ }^{2}$ Department of Information and Computer Science, Keio University, \\ 3-14-1 Hiyoshi, Kohoku-ku, Yokohama 223-8522, Japan \\ ${ }^{3}$ Geurdes Datascience, KvK 64522202, C vd Lijnstraat 164, 2593 NN, Den Haag Netherlands
}

(Dated: January 22, 2020)

Here, we consider the Bell experiment for a system described by multipartite states in the case where $n$-dichotomic observables are measured per site. If $n$ is two, we consider a two-setting Bell experiment. If $n$ is three, we consider a three-setting Bell experiment. Two-setting model is an explicit local realistic model for the values of a correlation function, given in a two-setting Bell experiment. Three-setting model is an explicit local realistic model for the values of a correlation function, given in a three-setting Bell experiment. In the non-contextual scenario, there is not the difference between three-setting model and two-setting model. And we cannot classify local realistic theories in this case. This says that we can construct three-setting model from two-setting model. Surprisingly we can discuss incompleteness in the Bell theorem using non-contextual model. On the other hand, in the contextual scenario, there is the difference between three-setting model and two-setting model. This says that we must distinguish three-setting model from two-setting model. And we can classify local realistic theories in this case.

PACS numbers: 03.65.Ud, 03.65.Ta, 03.65.Ca

Keywords: Quantum non locality, Quantum measurement theory, Formalism 


\section{INTRODUCTION}

Quantum mechanics (cf. [1 [ [ [ ] ] ) gives accurate and at times remarkably accurate numerical predictions for microscopic physical phenomena.

From the incompleteness argument of Einstein, Podolsky, and Rosen (EPR) 7], a hidden-variable interpretation of quantum mechanics is a topic of research [3, 4]. One is the Bell-EPR theorem [ 8 ]. This theorem says that the quantum predictions violate the inequality following from the EPR-locality condition. The locality condition says that a result of measurement pertaining to a system is independent of any measurement performed simultaneously at a distance on another system. Quantum mechanics does not allow a local realistic interpretation. Certain quantum predictions violate Bell inequalities [8], which are conditions that a local realistic theory must satisfy. Experimental efforts (Bell experiment) of a violation of local realism can be seen in [9 11]. Other types of inequalities are given in [12, 13]. Bell inequalities with settings other than spin polarizations can be seen [14].

Here, we consider the Bell experiment for a system described by multipartite states in the case where $n$-dichotomic observables are measured per site. If $n$ is two, we consider a two-setting Bell experiment. If $n$ is three, we consider a three-setting Bell experiment. Two-setting model is an explicit local realistic model for the values of a correlation function, given in a two-setting Bell experiment. Three-setting model is an explicit local realistic model for the values of a correlation function, given in a three-setting Bell experiment.

Classification of local realistic theories is discussed [15, 16]. We discuss two-setting model cannot construct threesetting model. The two models are different from each other. Clearly the argumentations rely on contextual local realistic models. However, if we take non-contextual local realistic models, then the situation changes. For example, we may accept Malley's supposition [17], that is, we suppose all quantum observables in a hidden-variable model must commute simultaneously. This is notable example for non-contextual scenario.

In the non-contextual local realistic model, there is not the difference between three-setting model and two-setting model. This says that we can construct three-setting model from two-setting model. Surprisingly we can discuss incompleteness (cf. [18]) in the Bell theorem by using non-contextual model.

We suppose the Bell theorem as follows [16]:

$$
\left(E_{\mathrm{LR}}, E\right)=(E, E)
$$

In this paper, our aim is of showing

$$
\left(E_{\mathrm{LR}}, E\right)<(E, E) .
$$

by using non-contextual local realistic model. Therefore, we discuss incompleteness in the Bell theorem by using non-contextual local realistic model.

On the other hand, in the contextual scenario, there must be the difference between three-setting model and twosetting model. This says that we must distinguish three-setting model from two-setting model. And we can classify local realistic theories in this case.

\section{INCOMPLETENESS IN THE BELL THEOREM USING NON-CONTEXTUAL LOCAL REALISTIC MODEL}

Assume that we have a set of $N$ spins $\frac{1}{2}$. Each of them is in a separate laboratory. As is well known the measurements (observables) for such spins are parameterized by a unit vector $\vec{n}_{j}, j=1,2, \ldots, N$ (its direction along which the spin component is measured). The results of measurements are \pm 1 (in $\hbar / 2$ unit). We can introduce the "Bell" correlation function, which is the average of the product of the local results:

$$
E\left(\vec{n}_{1}, \vec{n}_{2}, \ldots, \vec{n}_{N}\right)=\left\langle r_{1}\left(\vec{n}_{1}\right) r_{2}\left(\vec{n}_{2}\right) \cdots r_{N}\left(\vec{n}_{N}\right)\right\rangle_{\mathrm{avg}}
$$

where $r_{j}\left(\vec{n}_{j}\right)$ is the local result, \pm 1 , which is obtained if the measurement direction is set at $\vec{n}_{j}$.

If an experimental correlation function admits rotationally invariant tensor structure familiar from Newton's theory, we can introduce the following form:

$$
E\left(\vec{n}_{1}, \vec{n}_{2}, \ldots, \vec{n}_{N}\right)=\hat{T} \cdot\left(\vec{n}_{1} \otimes \vec{n}_{2} \otimes \cdots \otimes \vec{n}_{N}\right)
$$

where $\otimes$ denotes the tensor product, $\cdot$ denotes the scalar product in $\mathrm{R}^{3 \mathrm{~N}}$, and $\hat{T}$ is the correlation tensor given by

$$
T_{i_{1} \ldots i_{N}} \equiv E\left(\vec{x}_{1}^{\left(i_{1}\right)}, \vec{x}_{2}^{\left(i_{2}\right)}, \ldots, \vec{x}_{N}^{\left(i_{N}\right)}\right)
$$


where $\vec{x}_{j}^{\left(i_{j}\right)}$ is a unit directional vector of the local coordinate system of the $j$ th observer; $i_{j}=1,2,3$ gives the full set of orthogonal vectors defining the local Cartesian coordinates. Obviously the assumed form of (4) implies rotational invariance, because the correlation function does not depend on the coordinate systems used by the observers. Rotational invariance simply states that the value of $E\left(\vec{n}_{1}, \vec{n}_{2}, \ldots, \vec{n}_{N}\right)$ cannot depend on the local coordinate systems used by the $N$ observers.

Assume that one knows the values of all $3^{N}$ components of the correlation tensor, $T_{i_{1} \ldots i_{N}}$, which are obtainable by performing specific $3^{N}$ measurements of the correlation function, (cf. Eq. (5)). Then, with the use of the formula (41) we can reproduce the values of the correlation functions for all other possible sets of local settings. Using this rotationally invariant structure of the correlation function, we shall derive a necessary condition for the existence of a local realistic theory of the experimental correlation function given in (44). If the correlation function is described by the local realistic theory, then the correlation function must be simulated by the following structure

$$
E_{\mathrm{LR}}\left(\vec{n}_{1}, \vec{n}_{2}, \ldots, \vec{n}_{N}\right)=\int d \lambda \rho(\lambda) I^{(1)}\left(\vec{n}_{1}, \lambda\right) I^{(2)}\left(\vec{n}_{2}, \lambda\right) \cdots I^{(N)}\left(\vec{n}_{N}, \lambda\right),
$$

where $\lambda$ is some local hidden variable, $\rho(\lambda)$ is a probabilistic distribution, and $I^{(j)}\left(\vec{n}_{j}, \lambda\right)$ is the predetermined "hidden" result of the measurement of the dichotomic observable $\vec{n} \cdot \sigma$ with values \pm 1 .

Let us parametrize the three unit vectors in the plane defined $\vec{x}_{j}^{(1)}$ and $\vec{x}_{j}^{(2)}$ in the following way:

$$
\vec{n}_{j}\left(\alpha_{j}^{l_{j}}\right)=\cos \alpha_{j}^{l_{j}} \vec{x}_{j}^{(1)}+\sin \alpha_{j}^{l_{j}} \vec{x}_{j}^{(2)}, \quad j=1,2, \ldots, N .
$$

The phases $\alpha_{j}^{l_{j}}$ that experimentalists are allowed to set are chosen as:

$$
\alpha_{j}^{l_{j}}=\left(l_{j}-1\right) \pi / 3, \quad l_{j}=1,2,3 .
$$

We shall show that scalar product of the local realistic correlation function

$$
E_{\mathrm{LR}}\left(\alpha_{1}^{l_{1}}, \alpha_{2}^{l_{2}}, \ldots, \alpha_{N}^{l_{N}}\right)=\int d \lambda \rho(\lambda) I^{(1)}\left(\alpha_{1}^{l_{1}}, \lambda\right) I^{(2)}\left(\alpha_{2}^{l_{2}}, \lambda\right) \cdots I^{(N)}\left(\alpha_{N}^{l_{N}}, \lambda\right),
$$

with the rotationally invariant correlation function, that is,

$$
E\left(\alpha_{1}^{l_{1}}, \alpha_{2}^{l_{2}}, \ldots, \alpha_{N}^{l_{N}}\right)=\hat{T} \cdot\left(\vec{n}_{1}\left(\alpha_{1}^{l_{1}}\right) \otimes \vec{n}_{2}\left(\alpha_{2}^{l_{2}}\right) \otimes \cdots \otimes \vec{n}_{N}\left(\alpha_{N}^{l_{N}}\right)\right),
$$

is bounded by a specific number dependent on $\hat{T}$. We define the scalar product $\left(E_{\mathrm{LR}}, E\right)$ as follows: We see that the maximal possible value of $\left(E_{\mathrm{LR}}, E\right)$ is bounded as:

$$
\left(E_{\mathrm{LR}}, E\right)=\sum_{l_{1}=1,2,3} \sum_{l_{2}=1,2,3} \cdots \sum_{l_{N}=1,2,3} E_{\mathrm{LR}}\left(\alpha_{1}^{l_{1}}, \alpha_{2}^{l_{2}}, \ldots, \alpha_{N}^{l_{N}}\right) E\left(\alpha_{1}^{l_{1}}, \alpha_{2}^{l_{2}}, \ldots, \alpha_{N}^{l_{N}}\right) \leq 2^{N} T_{\max }
$$

where $T_{\max }$ is the maximal possible value of the correlation tensor component, i.e.,

$$
T_{\max }=\max _{\beta_{1}, \beta_{2}, \ldots, \beta_{N}} E\left(\beta_{1}, \beta_{2}, \ldots, \beta_{N}\right),
$$

where $\beta_{j}$ is some angle.

A necessary condition for the existence of the local realistic description $E_{\mathrm{LR}}$ of the experimental correlation function

$$
E\left(\alpha_{1}^{l_{1}}, \alpha_{2}^{l_{2}}, \ldots, \alpha_{N}^{l_{N}}\right)=E\left(\vec{n}_{1}\left(\alpha_{1}^{l_{1}}\right), \vec{n}_{2}\left(\alpha_{2}^{l_{2}}\right), \ldots, \vec{n}_{N}\left(\alpha_{N}^{l_{N}}\right)\right)
$$

that is for $E_{\mathrm{LR}}$ to be equal to $E$ for the three measurement directions, is that one has $\left(E_{\mathrm{LR}}, E\right)=(E, E)$. This implies the possibility of modeling $E$ by the three-setting local realistic correlation function $E_{\mathrm{LR}}$ given in (9) with respect to the three measurement directions. If we have $\left(E_{\mathrm{LR}}, E\right)<(E, E)$, then the experimental correlation function cannot be explainable by the three-setting local realistic model. (Note that, due to the summation in (11), we are looking for the three-setting model.)

In what follows, we derive the upper bound (11). Since the local realistic model is an average over $\lambda$, it is enough to find the bound of the following expression:

$$
\sum_{l_{1}=1,2,3} \cdots \sum_{l_{N}=1,2,3} I^{(1)}\left(\alpha_{1}^{l_{1}}, \lambda\right) \cdots I^{(N)}\left(\alpha_{N}^{l_{N}}, \lambda\right) \sum_{i_{1}, i_{2}, \ldots, i_{N}=1,2} T_{i_{1} i_{2} \ldots i_{N}} c_{1}^{i_{1}} c_{2}^{i_{2}} \cdots c_{N}^{i_{N}}
$$

where

$$
\left(c_{j}^{1}, c_{j}^{2}\right)=\left(\cos \alpha_{j}^{l_{j}}, \sin \alpha_{j}^{l_{j}}\right),
$$


and

$$
T_{i_{1} i_{2} \ldots i_{N}}=\hat{T} \cdot\left(\vec{x}_{1}^{\left(i_{1}\right)} \otimes \vec{x}_{2}^{\left(i_{2}\right)} \otimes \cdots \otimes \vec{x}_{N}^{\left(i_{N}\right)}\right),
$$

compare (4) and (5).

Let us analyze the structure of the sum (14). Note that (14) is a sum, with coefficients given by $T_{i_{1} i_{2} \ldots i_{N}}$, which is a product of the following sums:

$$
\sum_{l_{j}=1,2,3} I^{(j)}\left(\alpha_{j}^{l_{j}}, \lambda\right) \cos \alpha_{j}^{l_{j}}
$$

and

$$
\sum_{l_{j}=1,2,3} I^{(j)}\left(\alpha_{j}^{l_{j}}, \lambda\right) \sin \alpha_{j}^{l_{j}}
$$

We deal here with sums, or rather scalar products of $I^{(j)}\left(\alpha_{j}^{l_{j}}, \lambda\right)$ with two-orthogonal vectors. Ohe has

$$
\sum_{l_{j}=1,2,3} \cos \alpha_{j}^{l_{j}} \sin \alpha_{j}^{l_{j}}=0
$$

because

$$
2 \times \sum_{l_{j}=1,2,3} \cos \alpha_{j}^{l_{j}} \sin \alpha_{j}^{l_{j}}=\sum_{l_{j}=1,2,3} \sin 2 \alpha_{j}^{l_{j}}=\operatorname{Im}\left(\sum_{l_{j}=1,2,3} e^{i 2 \alpha_{j}^{l_{j}}}\right) .
$$

Since $\sum_{l_{j}=1}^{3} e^{i\left(l_{j}-1\right)(2 / 3) \pi}=0$, the last term vanishes.

Please note

$$
\sum_{l_{j}=1}^{3}\left(\cos \alpha_{j}^{l_{j}}\right)^{2}=\sum_{l_{j}=1}^{3} \frac{1+\cos 2 \alpha_{j}^{l_{j}}}{2}=3 / 2
$$

and

$$
\sum_{l_{j}=1}^{3}\left(\sin \alpha_{j}^{l_{j}}\right)^{2}=\sum_{l_{j}=1}^{3} \frac{1-\cos 2 \alpha_{j}^{l_{j}}}{2}=3 / 2
$$

because,

$$
\sum_{l_{j}=1,2,3} \cos 2 \alpha_{j}^{l_{j}}=\operatorname{Re}\left(\sum_{l_{j}=1,2,3} e^{i 2 \alpha_{j}^{l_{j}}}\right) .
$$

Since $\sum_{l_{j}=1}^{3} e^{i\left(l_{j}-1\right)(2 / 3) \pi}=0$, the last term vanishes.

The normalized vectors $M_{1} \equiv \sqrt{\frac{2}{3}}(\cos 0, \cos \pi / 3, \cos 2 \pi / 3)$ and $M_{2} \equiv \sqrt{\frac{2}{3}}(\sin 0, \sin \pi / 3, \sin 2 \pi / 3)$ form a basis of a real two-dimensional plane, which we shall call $S^{(2)}$. Note further that any vector in $S^{(2)}$ is of the form:

$$
A \cdot M_{1}+B \cdot M_{2},
$$

where $A$ and $B$ are constants, and that any normalized vector in $S^{(2)}$ is given by

$$
\cos \psi M_{1}+\sin \psi M_{2}=\sqrt{\frac{2}{3}}(\cos (0-\psi), \cos (\pi / 3-\psi), \cos (2 \pi / 3-\psi)) .
$$

The norm $\left\|I^{(j) \|}\right\|$ of the projection of $I^{(j)}$ into the plane $S^{(2)}$ is given by the maximal possible value of the scalar product $I^{(j)}$ with any normalized vector belonging to $S^{(2)}$, that is

$$
\left\|I^{(j) \|}\right\|=\max _{\psi} \sum_{l_{j}=1,2,3} I^{(j)}\left(\alpha_{j}^{l_{j}}, \lambda\right) \sqrt{\frac{2}{3}} \cos \left(\alpha_{j}^{l_{j}}-\psi\right)=\sqrt{\frac{2}{3}} \max _{\psi} \operatorname{Re}(z \exp (i(-\psi))),
$$


where $z=\sum_{l_{j}=1}^{3} I^{(j)}\left(\alpha_{j}^{l_{j}}, \lambda\right) \exp \left(i \alpha_{j}^{l_{j}}\right)$. We may assume $\left|I^{(j)}\left(\alpha_{j}^{l_{j}}, \lambda\right)\right|=1$. Then, since $e^{i \alpha_{j}^{l_{j}}}=e^{i\left[\left(l_{j}-1\right) / 3\right] \pi}$, the possible values for $z$ are $0, \pm 2 e^{i(\pi / 3)}, \pm 2 e^{i(2 \pi / 3)}, \pm 2$. Note that the minimum possible overall complex phase (modulo $2 \pi$ ) of $z \exp (i(-\psi))$ is 0 . Then we obtain $\left\|I^{(j) \|}\right\| \leq \sqrt{\frac{2}{3}} \times 2 \cos 0=2 \sqrt{\frac{2}{3}}$.

Since $M_{1}$ and $M_{2}$ are two-orthogonal basis vectors in $S^{(2)}$, one has

$$
\sum_{l_{j}=1,2,3} I^{(j)}\left(\alpha_{j}^{l_{j}}, \lambda\right) \cdot \sqrt{\frac{2}{3}} \cos \alpha_{j}^{l_{j}}=\cos \beta_{j}\left\|I^{(j)}\right\| \|,
$$

and

$$
\sum_{l_{j}=1,2,3} I^{(j)}\left(\alpha_{j}^{l_{j}}, \lambda\right) \cdot \sqrt{\frac{2}{3}} \sin \alpha_{j}^{l_{j}}=\sin \beta_{j}\left\|I^{(j) \|}\right\|,
$$

where $\beta_{j}$ is some angle. Using this fact one can put the value of (14) into the following form:

$$
\left(\sqrt{\frac{3}{2}}\right)^{N} \prod_{j=1}^{N}\left\|I^{(j) \|}\right\| \times \sum_{i_{1}, i_{2}, \ldots, i_{N}=1,2} T_{i_{1} i_{2} \ldots i_{N}} d_{1}^{i_{1}} d_{2}^{i_{2}} \cdots d_{N}^{i_{N}}
$$

where

$$
\left(d_{j}^{1}, d_{j}^{2}\right)=\left(\cos \beta_{j}, \sin \beta_{j}\right)
$$

Let us look at the expression

$$
\sum_{i_{1}, i_{2}, \ldots, i_{N}=1,2} T_{i_{1} i_{2} \ldots i_{N}} d_{1}^{i_{1}} d_{2}^{i_{2}} \cdots d_{N}^{i_{N}}
$$

Formula (30) shows that we deal here with two-dimensional unit vectors $\vec{d}_{j}=\left(d_{j}^{1}, d_{j}^{2}\right), j=1,2, \ldots, N$, therefore (31) is equal to $\hat{T} \cdot\left(\overrightarrow{d_{1}} \otimes \vec{d}_{2} \otimes \cdots \otimes \vec{d}_{N}\right)$, i.e., it is a component of the tensor $\hat{T}$ in the directions specified by the vectors $\vec{d}_{j}$. If one knows all the values of $T_{i_{1} i_{2} \ldots i_{N}}$, one can always find the maximal possible value of such a component, and it is equal to $T_{\max }$, of equation (12).

Therefore since $\left\|I^{(j) \|}\right\| \leq 2 \sqrt{\frac{2}{3}}$ the maximal value of (29) is $2^{N} T_{\max }$, and finally one has

$$
\left(E_{\mathrm{LR}}, E\right) \leq 2^{N} T_{\max }
$$

Please note that relation (32) is a generalized Bell inequality. Specific local realistic models, which predict threesetting models, must satisfy it. In the following, we shall show that if one replaces $E_{\mathrm{LR}}$ by $E$ one may have a violation of the inequality (32). One has

$$
(E, E)=\sum_{l_{1}=1,2,3} \sum_{l_{2}=1,2,3} \ldots \sum_{l_{N}=1,2,3}\left(\sum_{i_{1}, i_{2}, \ldots, i_{N}=1,2} T_{i_{1} i_{2} \ldots i_{N}} c_{1}^{i_{1}} c_{2}^{i_{2}} \cdots c_{N}^{i_{N}}\right)^{2}=\left(\frac{3}{2}\right)^{N} \sum_{i_{1}, i_{2}, \ldots, i_{N}=1,2} T_{i_{1} i_{2} \ldots i_{N}}^{2} .
$$

Here, we use the fact that $\sum_{l_{j}=1,2,3} c_{j}^{\alpha} c_{j}^{\beta}=\frac{3}{2} \delta_{\alpha, \beta}$, because $c_{j}^{1}=\cos \alpha_{j}^{l_{j}}$ and $c_{j}^{2}=\sin \alpha_{j}^{l_{j}}$.

The structure of condition (32) and the value (33) suggests that the value of (33) does not have to be smaller than (32). That is there may be such correlation functions $E$, which have the property that for any $E_{\text {LR }}$ (three-setting model) one has $\left(E_{\mathrm{LR}}, E\right)<(E, E)$, which implies impossibility of modeling $E$ by the three-setting local realistic correlation function $E_{\mathrm{LR}}$ with respect to the three measurement directions.

We shall present an important quantum state. Consider the following $N$-qubit Greenberger-Horne-Zeilinger (GHZ) state [19]

$$
|\psi\rangle=\frac{1}{\sqrt{2}}\left(|z+\rangle_{1} \cdots|z+\rangle_{N}+|z-\rangle_{1} \cdots|z-\rangle_{N}\right)
$$

where $|z \pm\rangle_{j}$ is the eigenstate of the local $\sigma_{z}$ operator of the $j$ th observer. We introduce a mixture of GreenbergerHorne-Zeilinger correlations and white noise:

$$
\rho=V|\psi\rangle\langle\psi|+(1-V) \rho_{\text {noise }}
$$


where $|\psi\rangle$ is the GHZ state and $\rho_{\text {noise }}=\frac{1}{2^{N}} I$ is the random noise admixture. The value of $V$ can be interpreted as the reduction factor of the interferometric contrast observed in the $N$-particle correlation experiment.

Imagine $N$ observers who can choose between two orthogonal directions of spin measurement, $\vec{x}_{j}^{(1)}$ and $\vec{x}_{j}^{(2)}$ for the $j$ th one. Let us assume that the source of $N$ entangled spin-carrying particles emits them in a state, which can be described as noisy Greenberger-Horne-Zeilinger correlations, given in (35). We can show that if the observers limit their settings to $\vec{x}_{j}^{(1)}=\hat{x}_{j}$ and $\vec{x}_{j}^{(2)}=\hat{y}_{j}$ there are

$$
2^{N}-1
$$

components of $\hat{T}$ of the value $\pm V$. These are $T_{11 \ldots 1}$ and all components that except from indices 1 have an even number of indices 2 . Other $x-y$ components vanish.

It is easy to see that $T_{\max }=V$ and $\sum_{i_{1}, i_{2}, \ldots, i_{N}=1,2} T_{i_{1} i_{2} \ldots i_{N}}^{2}=V^{2} 2^{N-1}$. Then we have $\left(E_{\mathrm{LR}}, E\right) \leq 2^{N} V$ and $(E, E)=\left(\frac{3}{2}\right)^{N} V^{2} 2^{N-1}=\frac{3^{N}}{2} V^{2}$. For $N \geq 6$ and $V$ given by

$$
2\left(\frac{2}{3}\right)^{N}<V \leq \frac{1}{\sqrt{2^{N-1}}}
$$

we see the fact that there exist two-setting local realistic models for three measurement directions $(A, B),(B, C),(C, A)$ in consideration $\left(\left(0, \frac{\pi}{3}, \frac{2 \pi}{3}\right) \equiv(A, B, C)\right)$ and these models $(A, B),(B, C),(C, A)$ can construct three-setting local realistic models $(A, B, C)$ because we suppose they are non-contextual local realistic models. And we have a violation of the Bell theorem:

$$
\left(E_{L R}, E\right)<(E, E) .
$$

That is, non-contextual local realistic models violate the Bell theorem.

As it is shown in [20] if the correlation tensor satisfies the following conditions

$$
\sum_{i_{1}, i_{2}, \ldots, i_{N}=1,2} T_{i_{1} i_{2} \ldots i_{N}}^{2} \leq 1,
$$

then there always exists non-contextual local realistic model for the set of correlation function values for all directions lying in a plane. For our example the condition (39) is met whenever $V \leq \frac{1}{\sqrt{2^{N-1}}}$. Nevertheless we have a violation of the Bell theorem for $V>2\left(\frac{2}{3}\right)^{N}$.

The situation is such that for $V \leq \frac{1}{\sqrt{2^{N-1}}}$ for all two settings per observer experiments we can construct a local realistic theory for the values of the correlation function for the settings chosen in the experiment. These theories must be consistent with each other if we want to extend their validity beyond the $2^{N}$ settings to which each of them pertains.

Here we suppose they are non-contextual local realistic models. Then we can extend their validity beyond the $2^{N}$ settings to which each of them pertains. Our calculations clearly indicates that this is possible for $V>2\left(\frac{2}{3}\right)^{N}$. And we have the violation of the Bell theorem for $V>2\left(\frac{2}{3}\right)^{N}$. Therefore, we discuss incompleteness in the Bell theorem using non-contextual local realistic model.

In the contextual scenario, there is the difference between three-setting model and two-setting model. This says that we must distinguish three-setting model from two-setting model. And we can classify local realistic theories in this case.

\section{CONCLUSIONS}

In conclusions, in the non-contextual scenario, there has not been the difference between three-setting model and two-setting model. And we cannot have classified local realistic theories in this case. This has said that we can construct three-setting model from two-setting model. Surprisingly we can have discussed incompleteness in the Bell theorem using non-contextual model. On the other hand, in the contextual scenario, there has been the difference between three-setting model and two-setting model. This has said that we must distinguish three-setting model from two-setting model. And we can have classified local realistic theories in this case.

\section{NOTE}

On behalf of all authors, the corresponding author states that there is no conflict of interest. 


\section{ACKNOWLEDGMENTS}

We thank Professor Do Ngoc Diep and Professor Germano Resconi for valuable comments.

[1] J. von Neumann, Mathematical Foundations of Quantum Mechanics (Princeton University Press, Princeton, New Jersey, 1955).

[2] R. P. Feynman, R. B. Leighton, and M. Sands, Lectures on Physics, Volume III, Quantum mechanics (Addison-Wesley Publishing Company, 1965).

[3] M. Redhead, Incompleteness, Nonlocality, and Realism (Clarendon Press, Oxford, 1989), 2nd ed.

[4] A. Peres, Quantum Theory: Concepts and Methods (Kluwer Academic, Dordrecht, The Netherlands, 1993).

[5] J. J. Sakurai, Modern Quantum Mechanics (Addison-Wesley Publishing Company, 1995), Revised ed.

[6] M. A. Nielsen and I. L. Chuang, Quantum Computation and Quantum Information (Cambridge University Press, 2000).

[7] A. Einstein, B. Podolsky, and N. Rosen, Phys. Rev. 47, 777 (1935).

[8] J. S. Bell, Physics 1, 195 (1964).

[9] A. Aspect, P. Grangier, and G. Roger, Phys. Rev. Lett. 47460 (1981).

[10] A. Aspect, P. Grangier, and G. Roger, Phys. Rev. Lett. 49, 91 (1982).

[11] A. Aspect, J. Dalibard, and G. Roger, Phys. Rev. Lett. 49, 1804 (1982).

[12] J. F. Clauser and M. A. Horne, Phys. Rev. D 10, 526 (1974).

[13] E. P. Wigner, Am. J. Phys. 38, 1005 (1970).

[14] A. Bramon and M. Nowakowski, Phys. Rev. Lett. 83, 1 (1999).

[15] K. Nagata, W. Laskowski, M. Wieśniak, and M. Żukowski, Phys. Rev. Lett. 93, 230403 (2004).

[16] K. Nagata, J. Phys. A: Math. Theor. 41, 155308 (2008).

[17] J. D. Malley, Phys. Rev. A 69, 022118 (2004).

[18] H. Geurdes, K. Nagata, T. Nakamura, and A. Farouk, arXiv:1704.00005v4 [physics.gen-ph] (2019).

[19] D. M. Greenberger, M. A. Horne, and A. Zeilinger, in Bell's Theorem, Quantum Theory and Conceptions of the Universe, edited by M. Kafatos (Kluwer Academic, Dordrecht, The Netherlands, 1989), p. 69.

[20] M. Żukowski and Č. Brukner, Phys. Rev. Lett. 88, 210401 (2002). 